\title{
Macular Pigment Optical Density Fluctuation as a Function of Pupillary Mydriasis: Methodological Considerations for Dual- Wavelength Autofluorescence
}

\author{
Ekaterina Loskutova \\ Technological University Dublin, ekaterina.loskutova@mydit.ie \\ John Butler \\ Technological University Dublin, john.s.butler@tudublin.ie \\ Gabriela Hernandez-Martinez \\ Technological University Dublin, gabriela.martinez@tudublin.ie
}

See next page for additional authors

Follow this and additional works at: https://arrow.tudublin.ie/otpomart

Part of the Optometry Commons

\section{Recommended Citation}

Loskutova, E., Butler, J.S. \& Hernnandez Martinez, G. (2020). Macular Pigment Optical Density Fluctuation as a Function of Pupillary Mydriasis: Methodological Considerations for Dual-Wavelength

Autofluorescence. Current Eye Research, vol. 46, no. 4. doi:10.1080/02713683.2020.1815792

This Article is brought to you for free and open access by ARROW@TU Dublin. It has been accepted for inclusion in Articles by an authorized administrator of ARROW@TU

Dublin. For more information, please contact arrow.admin@tudublin.ie, aisling.coyne@tudublin.ie, gerard.connolly@tudublin.ie.

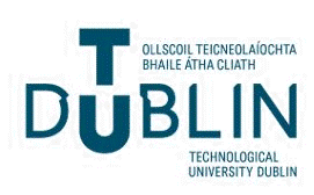


Authors

Ekaterina Loskutova, John Butler, Gabriela Hernandez-Martinez, Ian Flitcroft, and James Loughman

This article is available at ARROW@TU Dublin: https://arrow.tudublin.ie/otpomart/90 


\section{Macular Pigment Optical Density Fluctuation as a Function of Pupillary Mydriasis: Methodological Considerations for Dual-Wavelength Autofluorescence}

\section{Ekaterina Loskutova, John S. Butler, Gabriela Hernandez Martinez, Ian Flitcroft \& James Loughman}

To cite this article: Ekaterina Loskutova, John S. Butler, Gabriela Hernandez Martinez, lan Flitcroft \& James Loughman (2021) Macular Pigment Optical Density Fluctuation as a Function of Pupillary Mydriasis: Methodological Considerations for Dual-Wavelength Autofluorescence, Current Eye Research, 46:4, 532-538, DOI: 10.1080/02713683.2020.1815792

To link to this article: https://doi.org/10.1080/02713683.2020.1815792

\section{曲 Published online: 14 Sep 2020.}

\section{Submit your article to this journal $\llbracket$}

\section{Џ Article views: 56}

Q View related articles $₫$

View Crossmark data $₫$

Citing articles: 3 View citing articles ¿ð 


\title{
Macular Pigment Optical Density Fluctuation as a Function of Pupillary Mydriasis: Methodological Considerations for Dual-Wavelength Autofluorescence
}

\author{
Ekaterina Loskutova (iD) ${ }^{a}$, John S. Butler ${ }^{b}$, Gabriela Hernandez Martinez ${ }^{a}$, lan Flitcroft ${ }^{\mathrm{a}, \mathrm{c}}$, and James Loughman ${ }^{\mathrm{a}}$ \\ ${ }^{a}$ Centre for Eye Research Ireland, School of Physics, Clinical \& Optometric Sciences, Technological University Dublin, Dublin, Ireland; ${ }^{b} C e n t r e$ for Eye \\ Research Ireland, School of Mathematical Sciences, Technological University Dublin, Dublin, Ireland; 'Department of Ophthalmology, Children's \\ University Hospital, Dublin, Ireland
}

\begin{abstract}
Purpose of the study: Macular pigment (MP), comprising the dietary carotenoids lutein, zeaxanthin and meso-zeaxanthin, is believed to benefit eye health and vision. Numerous clinical and research devices and techniques are currently available to facilitate MP optical density (MPOD) measurement. One of those techniques, dual-wavelength fundus autofluorescence (AF) is being increasingly used for measurement of MP in the eye. There is substantial methodological variation across the published studies that have employed this technique, including in relation to the use of mydriasis, the possible influence of which does not appear to have been addressed in the literature. This prospective cross-sectional study was designed to investigate the effect of mydriasis on MP measurement quality and MPOD values obtained with dual-wavelength AF using the Heidelberg Spectralis HRA+OCT device.

Materials and Methods: Twenty-one healthy participants were recruited to the study. The mean age of participants was 44.8 years $( \pm 14.63)$. Pupil size and MPOD were measured in one eye for each participant, initially under natural pupil conditions and subsequently 30 minutes following instillation of one drop of $0.5 \%$ tropicamide.

Results: Despite providing MPOD measurements for the majority of undilated eyes (85.7\% of eyes herein), pupillary dilation resulted in statistically significant changes in MPOD ( $p<.001$ for central eccentricities). Our results indicate that the changes in MPOD were not uniform across the spatial profile. Marked improvements were also observed in image quality post-dilation $(p<.002$ for central eccentricities).

Conclusions: This study clearly demonstrates that dual-wavelength AF measurements of MPOD in the same eye vary as a function of pupillary dilation status, with MPOD under-estimated across the entire spatial profile of MP for natural relative to dilated pupillary conditions. Mydriasis should, therefore, be used routinely for MPOD measurements using dual wavelength $A F$, pupil size should be reported and image quality optimized in order to ensure accurate MPOD quantification.
\end{abstract}

ARTICLE HISTORY

Received 30 April 2020

Revised 10 August 2020

Accepted 17 August 2020

\section{KEYWORDS}

Macular pigment; dualwavelength

autofluorescence; mydriasis; spectralis; image quality

\section{Introduction}

Macular pigment (MP) is composed of the three dietary carotenoids: lutein $(\mathrm{L})$, zeaxanthin $(\mathrm{Z})$, and meso-zeaxanthin (MZ) which accumulate selectively in the retina, giving the macula lutea its characteristic yellow appearance. MP's constituent carotenoids possess short-wavelength light-filtering, antioxidant, anti-inflammatory, and neuro-protective properties, which are believed to benefit eye health and vision. ${ }^{1,2}$ $\mathrm{L}$ and $\mathrm{Z}$ are also found in several specific regions in the brain including the frontal, occipital, and temporal cortices, hippocampus, and cerebellum. ${ }^{3-6}$ Studies have demonstrated that MP can be augmented by supplementation or by incorporating more carotenoid-rich food into the diet. ${ }^{7,8} \mathrm{MP}$ and its constituent carotenoids have been studied extensively for their role in eye health and in the prevention of retinal disease such as agerelated macular degeneration and, more recently, conditions such as diabetes and glaucoma. ${ }^{9-11}$ There is also a limited body of research exploring the role of MP for cognitive health. ${ }^{12-14}$ To study the role of MP for ocular and cognitive health, a valid measure of MP in the eye is required.
Heterochromatic flicker photometry (HFP) is accepted as the gold standard for measurement of MPOD. ${ }^{15,16}$ However, HFP requires good vision and is reliant on participants to follow detailed instructions, sustain concentration, and fixation over an extended period. MPOD can, therefore, prove difficult to measure using the HFP technique, particularly in older participants with ophthalmic disease such as glaucoma. ${ }^{9,17}$ It also takes considerable time to obtain MP data at sufficient retinal eccentricities to build up a spatial profile of MPOD using HFP.

Dual-wavelength fundus autofluorescence (AF) is less widely used, but provides a non-psychophysical method of measuring MPOD based on detection of the fluorescence derived from lipofuscin. ${ }^{18}$ The technique requires minimal participation by the subject, who is required only to fixate on an internal target within the system for 30 seconds. Dualwavelength AF has been shown to achieve reliable and reproducible measures of MPOD. ${ }^{19,20}$ This technique provides an additional benefit as it measures the entire spatial profile (see Figure 1) and volume of MP (compared to the isolated single

CONTACT Ekaterina Loskutova ceri@tudublin.ie Centre for Eye Research Ireland, Environmental Sustainability and Health Institute, College of Sciences \& Health, School of Physics, Clinical and Optometric Sciences Technological University Dublin, Dublin, Ireland 


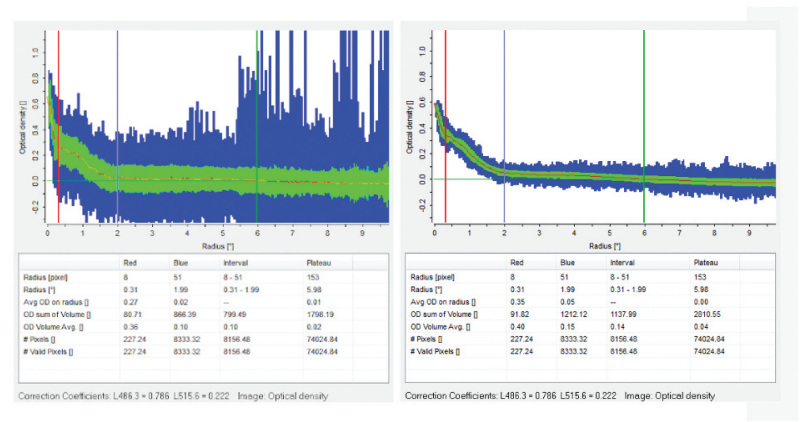

Figure 1. Example of MP Spatial profile output obtained with dual-wavelength autofluorescence using the Heidelberg Spectralis ${ }^{\circledast}$ HRA + OCT Multicolor (Heidelberg Engineering $\mathrm{GmbH}$, Heidelberg, Germany) for undilated (left) and dilated (right) conditions in the same eye. Note: retinal eccentricity is provided on the $\mathrm{X}$-axis and MPOD on the Y-axis. MPOD is indicated by the central dark thin line located in the middle of the green zone. The width of the green zone provides a visual indication of the standard deviation of the measures on MPOD at each eccentricity (no quantitative data are provided as to the size of the standard deviation). A wider green zone indicates greater standard deviation in the MPOD measurement, equating to a poorer quality measure. The blue bars above and below the green zone provide the minimum and maximum MPOD values measured at each eccentricity (again, no quantitative output is given as a measure of this minimum and maximum value, just the visual representation).

points measured by HFP). Although the device required for dual-wavelength fundus AF measurement is significantly more expensive than HFP, this technique provides more information regarding the complete spatial profile of MPOD at any single measurement, and enhanced capacity to detect any change across this profile in response to temporal variation in dietary intake, supplement use, or disease status.

Dual-wavelength AF measurements of MPOD in healthy participants have been shown to demonstrate good agreement with measurements using HFP, ${ }^{20}$ customized HFP, ${ }^{19}$ and a dual-wavelength reflectance technique. ${ }^{20}$ Additionally, MPOD measured with this method appears to correlate well with serum $\mathrm{L}$ and $\mathrm{Z}$, and with skin carotenoid levels. ${ }^{21}$ It has been previously reported, however, that cataract may result in underestimated MPOD when using $\mathrm{AF}^{22,23}$ so it would seem prudent to account for cataract status in dual-wavelength $\mathrm{AF}$ measurements of MPOD. Dual-wavelength AF has also been demonstrated to be capable of detecting change in MPOD following supplementation. ${ }^{24-26}$

The possible influence of pupil size on MPOD measurement using dual-wavelength AF does not appear to have been addressed in the literature. In most cases, it is possible to measure MPOD under natural un-dilated pupil conditions, even though the measurement requires exposure to an intense light source. Although pupillary dilation is generally accepted as a methodological requirement for MPOD measurement using dual-wavelength $\mathrm{AF}$, a review of the available literature reveals numerous published studies that do not report using mydriatic eye drops to dilate the pupil before MPOD measurement with this technique. ${ }^{24,27-31}$ Moreover, even in studies that report dilating for dual-wavelength fundus AF MPOD measurement, specific detail such as the actual pupil size following dilation is not typically provided. ${ }^{32-34}$ Importantly, only three of the (circa 100) studies that we reviewed, reported using a measure of MPOD image quality, a feature which is not provided inherently by the device. ${ }^{19,22,35}$

\section{Methods}

This prospective cross-sectional study was designed to investigate the effect of mydriasis on MP measurement quality and MPOD values obtained with dual-wavelength AF using the Heidelberg Spectralis HRA+OCT MultiColor (Heidelberg Engineering $\mathrm{GmbH}$, Germany).

Twenty-one healthy subjects without retinal pathology or cataract were recruited into the study. The mean age of participants was 44.8 years $( \pm 14.63)$, with a range from 27 to 70 years.

A comprehensive ocular examination was conducted to determine eye health, including a review of ocular and medical history, distance monocular visual acuity (VA) using ETDRS chart with participant's spectacles, slit lamp, and Volk lens examination, intraocular pressure measurement with the Icare ic100 tonometer, fundus imaging, and glaucoma module assessment using the Spectralis HRA+OCT device. The eye with better VA was selected as the study eye. All subjects had good vision, with a mean LogMAR visual acuity in the study eye of $-0.12( \pm 0.1)$ (Snellen equivalent $6 / 5+1)$.

The study was explained to each participant before obtaining written informed consent. The study was approved by the Research Ethics and Integrity Committee at Technological University Dublin (TU Dublin) and conformed to the tenets of the Declaration of Helsinki.

\section{Order of procedures}

First, minimum pupil size was measured using the Aladdin optical biometer (Topcon, Tokyo, Japan). Immediately after this, the first MPOD measurement was obtained using the Spectralis device. The study eye was then dilated with one drop of $0.5 \%$ tropicamide (Bausch + Lomb, Surrey, United Kingdom). After 30 minutes, a second set of pupillometry and MPOD measurements were captured. All measurements were obtained by the same examiner (EL).

\section{Minimum pupil size measurement}

The Aladdin biometer uses white light-emitting diodes (LEDs) to produce photopic light conditions and to constrict the pupil. Minimum pupil diameter was automatically measured using dynamic pupillometry. Pupil size was measured in a darkened room (lux levels at the eye when using the Aladdin device: $<0.01$ lux). Pupil size was recorded continuously for 20 seconds following exposure to a $0.4 \mathrm{~mW}$ bright LED flash. A graph of pupil size over time was plotted and minimum pupil size was recorded automatically as the lowest recorded size (typically reached within one to two secondspost flash).

\section{MPOD measurement}

MPOD was measured using a dual-wavelength AF method, as described previously, ${ }^{36,37}$ using the Heidelberg Spectralis. This device uses a confocal scanning laser ophthalmoscope (cSLO) and two excitation wavelengths (486 $\mathrm{nm}$ [blue light which is well absorbed by MP] and $516 \mathrm{~nm}$ [green light, absorption of 
which by MP is low]). The light intensity of the green and blue diode lasers in the Heidelberg Spectralis device is $0.44 \mathrm{~mW}$.

$\mathrm{AF}$ images of the $30^{\circ}$ central area of the retina were recorded for both wavelengths and foveal fluorescence was compared with a parafoveal reference point set at $6^{\circ}$ retinal eccentricity. MP density maps were then computed by the Heidelberg Eye Explorer software (HEYEX, version 1.9.13.0) by analyzing the AF images obtained at both wavelengths. As absorption of MP at $486 \mathrm{~nm}$ is high, and at $516 \mathrm{~nm}$ is close to zero, and there is virtually no $\mathrm{MP}$ in the parafoveal region that is used as a reference, total MP and its spatial profile could be determined (Figure 1).

MPOD measurements were obtained in a darkened room (lux levels at the eye when using the Spectralis device: 1 lux). After ensuring the correct positioning of the participant's head against the chin- and headrest, the AF mode was activated by switching the lever on the camera head to the vertical down position. The acquisition panel was then turned on, after which alignment, focus, and illumination were optimized in reflected infrared mode (IR). Then, IR mode was switched to blue AF + green $\mathrm{AF}$ (BAF+GAF) mode. Focus and image brightness were readjusted in the $B A F+G A F$ mode. Subjects were advised that they could blink if necessary. After at least 15 seconds of retinal bleaching, sequences of images were captured (recorded as a 30 second movie), which were then processed using an averaging technique by the Spectralis device. MP plots with MPOD values for circles centered on the fovea were automatically generated.

MPOD volume at $6^{\circ}$ retinal eccentricity as well as MPOD at $0.23,0.51,0.74,0.98,1.25,1.48,1.76,1.99,2.5,3.01$ degrees retinal eccentricity were obtained from the resulting MPOD plots. In addition, standard deviations (SDs) of MPOD at three eccentricities $(0.23,0.98$, and 1.99$)$ the green bars on Figure 1 were read from the plots using an open-source graph reader (graphreader.com). SDs at the three retinal eccentricities were used as an indicator of image quality.

Statistical analysis was performed using the SPSS statistical software package, V.24.0 (IBM-SPSS, Chicago, Illinois, USA). Figures were produced using the Microsoft R Studio package for Windows (R Core Team, 2019). ${ }^{38}$

\section{Results}

Undilated AF images with acceptable quality for MP calculation could be obtained for 18 subjects. In three subjects
(14.3\%), two attempts at capturing dual-wavelength AF video were made, but resulted in images that could not be processed by the device software. Successful measurements of MPOD were obtained for all 21 participants post-dilation. A marked improvement in image quality features (including SD, minimum and maximum values) was observed for every participant across the entire spatial profile of MP under dilated pupillary conditions (Figure 1).

Statistical analysis was performed on the 18 eyes of 18 subjects with available pre- and post-mydriasis MPOD and pupillometry measurements.

\section{MPOD, SD and minimum pupil size}

A paired samples t-test was conducted to compare MPOD volume, individual MPOD measurements at four commonly reported central eccentricities, minimum pupil size, and SD values at three selected eccentricities pre- and post-mydriasis. Pre-dilation measures of minimum pupil size, MPOD at all eccentricities, MPOD volume, and all SDs were statistically significantly different from those obtained post-dilation even after Bonferroni correction for multiple testing (pairedsamples t-test, $p \leq 0.002$ for all). Of note, $\mathrm{SD}$ values were substantially higher pre-mydriasis in every participant, with the mean SD between 2.2 and 2.8 times higher at the measured eccentricities under natural pupil conditions. The detailed preand post-dilation pupil size, SD, and MPOD data at commonly reported eccentricities are presented in Table 1. All data apart from SDs were normally distributed. To account for the nonnormal distribution of the SD data, Spearman's correlationranked order was used.

Figure 2 shows the spatial profile of mean MPOD for undilated (red) and dilated (green) pupils 30 minutes after instillation of tropicamide $0.5 \%$. A repeated measures mixed ANOVA was conducted including the factors dilation status (undilated and dilated) and eccentricities $\left(0.23^{\circ}, 0.51^{\circ}, 0.74^{\circ}, 0.98^{\circ}, 1.25^{\circ}\right.$, $\left.1.48^{\circ}, 1.76^{\circ}, 1.99^{\circ}, 2.5^{\circ}, 3.01^{\circ}\right)$. A statistically significant effect of dilation status $(\mathrm{F}(1,17)=73.134, p<.001)$ and eccentricity $(\mathrm{F}$ $(9,153)=163.619, p<.001)$ and a statistically significant interaction of dilation status and eccentricity $(F(9,153)=9.282$, $p<.001)$ was detected. Figure 2 illustrates the systematically higher MPOD values obtained following pupil dilation across the entire spatial profile of MP. The mean change in MPOD is not uniform however, with the highest level of change observed centrally ( $20 \%$ for the two most central eccentricities), while

Table 1. Minimum pupil size, MPOD, and SD data before and after mydriasis.

\begin{tabular}{|c|c|c|c|c|}
\hline Parameter & Undilated (mean \pm SD) & Dilated (mean \pm SD) & $\mathrm{t}(17)$ & $P$ value \\
\hline Minimum pupil size & $3.22 \pm 0.64$ & $5.5 \pm 0.88$ & -9.953 & $<0.001$ \\
\hline \multicolumn{5}{|l|}{ MPOD, mean } \\
\hline $0.23^{\circ}$ & $0.38 \pm 0.08$ & $0.46 \pm 0.08$ & -5.2 & $<0.001$ \\
\hline $0.51^{\circ}$ & $0.31 \pm 0.09$ & $0.37 \pm 0.1$ & -5.477 & $<0.001$ \\
\hline $0.74^{\circ}$ & $0.32 \pm 0.09$ & $0.37 \pm 0.09$ & -4.593 & $<0.001$ \\
\hline $0.98^{\circ}$ & $0.28 \pm 0.08$ & $0.31 \pm 0.08$ & -5.394 & $<0.001$ \\
\hline Volume, at $6^{\circ}$ & $3928.29 \pm 2035.65$ & $4884.43 \pm 2133.28$ & -11.529 & $<0.001$ \\
\hline MPOD plot SD $0.23^{\dagger}$ & $0.17 \pm 0.1$ & $0.06 \pm 0.02$ & 4.811 & $<0.001$ \\
\hline MPOD plot SD $0.98^{\dagger}$ & $0.13 \pm 0.07$ & $0.06 \pm 0.03$ & 3.908 & $<0.001$ \\
\hline MPOD plot SD $1.99^{\dagger}$ & $0.11 \pm 0.07$ & $0.04 \pm 0.02$ & 4.682 & $<0.001$ \\
\hline
\end{tabular}

$P$-value obtained using the paired samples t-test and a Wilcox Rank Sum test for the SD data. Minimum pupil size under bright illumination (light intensity $0.4 \mathrm{~mW}$ ). ${ }^{\dagger} \mathrm{SD}$ - standard deviation as derived from the green bars on MPOD plots. 


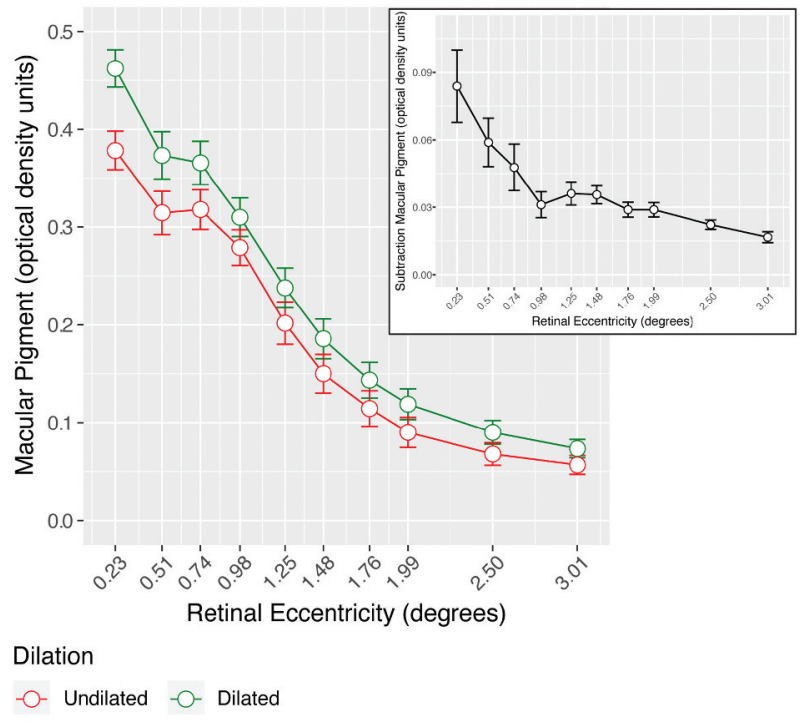

Figure 2. Mean and standard error of the spatial profile of MPOD across the central $3^{\circ}$, for undilated (red) and dilated (green) 30 minutes after instillation of tropicamide $0.5 \%$. Insert - Subtraction of undilated from dilated MPOD at each retinal eccentricity.

the difference is relatively consistently lower at more peripheral eccentricities. The statistically significant interaction between dilation and eccentricity is illustrated in the plot of the subtraction of dilated and undilated MPOD at each retinal eccentricity (insert of Figure 2).

\section{Image quality and its relationship to pupil size}

Figure 3 illustrates a marked reduction and compression of MPOD SD values, reflecting the improvement in image quality of the MPOD measurement following dilation for three indicative retinal eccentricities $\left(0.23^{\circ}, 0.98^{\circ}\right.$ and $\left.1.99^{\circ}\right)$.

To investigate the effect of dilation and eccentricity on the SD of MPOD, a repeated measures mixed ANOVA was conducted with the factors being dilation status (undilated, dilated) and eccentricities $(0.23,0.98,1.99)$. A statistically significant effect of dilation status $(\mathrm{F}(1,17)=18.114, p=.001)$ and eccentricity $(\mathrm{F}$
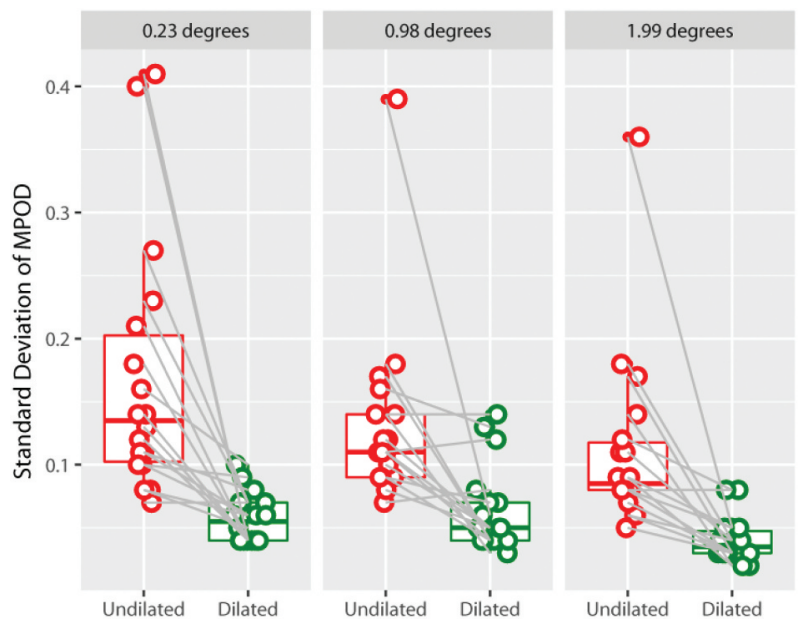

Figure 3. Boxplot and individual subject data of standard deviation of MPOD measurement at $0.23^{\circ}, 0.98^{\circ}$ and $1.99^{\circ}$ for undilated (red) and dilated (green) measurements.
$(2,34)=15.859, p<.001)$ and an interaction of dilation status and eccentricity was observed $(\mathrm{F}=8.395, p=.009)$.

A linear regression was calculated to investigate the SD measures obtained from the MP graph at $0.23^{\circ}$ retinal eccentricity as a function of pupil size and to determine a cut-off value for acceptable MPOD measurement quality. The simple linear regression showed a significant relationship between SD measures obtained from the MP graph at $0.23^{\circ}$ retinal eccentricity as a function of minimum pupil size $(\mathrm{F}(1,34)=15.64, p<.001)$. The slope coefficient for pupil size was $-0.03711(\mathrm{t}(34)=-3.995$, $p<.001)$ with the intercept of 0.274813 (t $(34)=6.412, p<.001$ ). The $\mathrm{R}^{2}$ value was 0.3151 so $31.5 \%$ of the variation in the SD of MPOD was explained by the model containing pupil size. This model gives the formula of $(\mathrm{SD}$ of MPOD $)=-0.037$ (Pupil size $)+$ 0.274813 .

An unsupervised k-means cluster analysis was conducted to categorize two clusters by minimum pupil size and SD measures obtained from the MP graph at $0.23^{\circ}$ retinal eccentricity. A scatter plot of each subjects SD of MPOD at $0.23^{\circ}$ as a function of pupil size and dilation status was produced with subject data assigned to a classification cluster. The analysis resulted in the classification of two equal groups of 18 , the first cluster contained 17 of the 18 participants post-dilation with 1 participant misclassified. In the second cluster, 17 of the 18 participants were classified as undilated with 1 participant misclassified (Figure 4). The unsupervised analysis resulted in a $94.4 \%$ accuracy of classification. The pupil size of $4.35 \mathrm{~mm}$ indicated by the vertical-dashed blue line which corresponds to the midpoint between cluster 1 and cluster 2 (Figure 4). Using the linear regression equation derived above, a $4.35 \mathrm{~mm}$ pupil size yields a predicted SD value of 0.11 which could be used as a cut-off value for acceptable MPOD measurement quality.

\section{Discussion}

This study provides comprehensive evidence that pupil size is an important consideration in the measurement of MP using

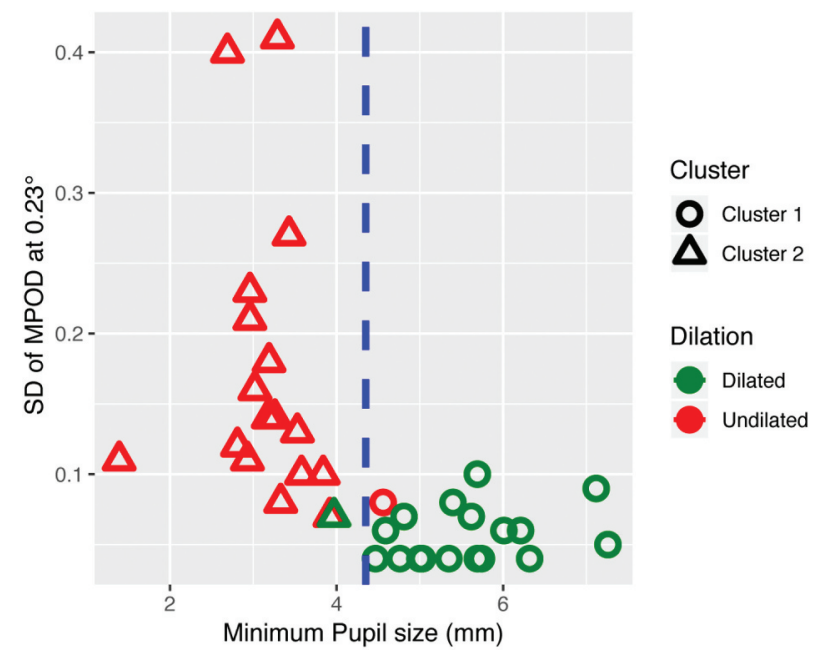

Figure 4. Scatter plot showing relationship between minimum pupil size and SD of the MPOD at $0.23^{\circ}$ retinal eccentricity. Green (dilated) and red (undilated) colors indicate pupil dilation status for each subject; blue line indicates the bisection line; the triangle and circle symbols indicated which k-means classification cluster the subject data was assigned to. 
the Spectralis dual-wavelength fundus AF device. Despite providing MPOD measurements for the majority of undilated eyes ( $85.7 \%$ of eyes herein), two critical observations can be made in relation to the impact of mydriasis. First, larger pupils facilitate better quality MPOD measures, evidenced by the tighter SDs (note the shorter green bars visible in Figure 1) and narrower range (note the variation in blue bars in Figure 1) observed post-dilation. This improvement in image quality and capacity to measure MPOD is not surprising given that the intensity of the stimuli used would cause a strong miotic response under natural pupillary conditions. Second, and perhaps more importantly, MPOD measurements in the same eye vary as a function of pupillary dilation status, with MPOD invariably under-estimated across the entire spatial profile of MP for natural relative to dilated pupillary conditions.

Dual-wavelength fundus AF is being increasingly used for the determination of ocular MPOD levels as it offers distinct benefits over other techniques. The time required to capture MPOD measurements is substantially reduced compared to psychophysical techniques so participant fatigue is no longer a measurement consideration. ${ }^{39}$ Additionally, it provides much more detailed information on the spatial profile of MP and the graphical output is clinically useful, ${ }^{40}$ particularly in relation to the evaluation of MPOD change over time. Of note, although Spectralis gathers clinically useful information on the entire special profile of MP, the density of carotenoid deposits decreases rapidly at extrafoveal eccentricities, which leads to increased variability in peripheral MP measures. Analysis of individual data points should, therefore, prioritize central MP measures including for the assessment of quality indices.

The measurements have proven to be relatively robust in terms of reproducibility over time in the absence of cataract. ${ }^{20}$ Good within-session repeatability has also been shown, although a higher coefficient of repeatability for MPOD measures inside $0.1^{\circ}$ of retinal eccentricity has prompted the suggestion to capture multiple MPOD measures to ensure robust values. ${ }^{41}$ Dual-wavelength AF devices used to measure MPOD are not generally commercially available to clinicians, so there are no practical guidelines available regarding a standardized method of measurement. Additionally, although the Heidelberg Spectralis provides a detailed graphical representation of the MPOD measurement, it does not provide any quantitative index of image quality or measurement acceptability. Although the differences in Figures 1 and 3 are marked, there is nothing in the measurement output to indicate at what point an acceptable level of quality has been achieved. Given the variation in MPOD values obtained under the two conditions, analysis of the graphical outputs to determine measurement acceptability would seem prudent.

In a clinical environment, it would be highly beneficial to avoid the necessity for dilation. Time and cost savings would benefit practitioners, patients would save time too and would avoid the short-term quality of life impact of dilation on visual comfort and performance. It could be argued that these benefits might outweigh a slight underestimation of MPOD. The level of underestimation in natural pupillary conditions was not consistent, however, either between participants or across the spatial profile of MP within participants. The difference in MPOD volume following dilation, for example, ranged between 263.61 and 1523.54 units of optical density volume. Moreover, the profile of MP overall was also different in preand post-mydriasis measurements. As illustrated in Figure 2, the profile of MP for undilated measurements appears flattened centrally relative to the periphery, not showing the full extent of the peak MPOD as observed in dilated conditions. The statistically significant interaction between dilation and eccentricity as illustrated on the insert in Figure 2 would suggest that any correction factor would need to incorporate retinal eccentricity as a means to adjust MPOD based on undilated measurements.

From a research perspective, our analysis of the methodological approach adopted in the published literature involving dual-wavelength AF for MPOD measurement reveals substantial variation between studies and, in many cases, insufficient detail to determine the validity of the MPOD findings. The lack of standardized approach to the measurement creates difficulty in relation to the interpretation of the relative findings reported across the various studies and may even invalidate the outcomes of some.

This is not the first study to demonstrate differences in MPOD measurements associated with the quality of measurement taken. The presence of cataract has also been shown to cause an underestimation of MPOD when measured using dual-wavelength AF by comparing MPOD levels pre- and postcataract surgery. ${ }^{22}$ It appears that the magnitude of difference in MP values was much higher (approximately 5 times higher) between then pre- and post-cataract measurements ( 0.26 optical density units at $0.51^{\circ}$ retinal eccentricity and 4.452 optical density units for volume at $8.98^{\circ}$ ) when compared to the preand post-mydriasis measurements in the current study $(0.059$ optical density units at $0.51^{\circ}$ retinal eccentricity and 956.14 optical density units for volume at $8.98^{\circ}$ ). Also baseline measurements could not be obtained for a higher percentage of participants in the cataract study (33\% compared to $14.3 \%$ in our study). This suggests that the effect of cataract on measurement is a lot more pronounced than that of the pupil size. Statistically significant variation of MP, however, was observed through all of the central eccentricities analyzed. The image quality index (IQI) calculated in the cataract study is very specific to cataract and therefore not useful in its current format for more general use. ${ }^{22}$

The protocol adopted herein, to measure MPOD 30 minutes after instillation of $0.5 \%$ tropicamide, was sufficient to allow a high degree of classification accuracy in our cluster analysis without any other assumptions. Dilated pupils tended to be larger than $4 \mathrm{~mm}$ under bright light stimulation (in $96 \%$ of cases, with the other one case just below $4 \mathrm{~mm}$ ), which delivered statistically significantly better image quality according to the computed SDs (mean SD values for dilated pupils ranged from 0.04 to 0.1 ). The $4 \mathrm{~mm}$ finding is entirely consistent with the optical set up of the dual-wavelength AF device. The incident blue and green laser beams intercept the pupil plane in a 1-2 mm diameter spot. The emitted AF is captured by the entrance pupil (detection system), which has a diameter of $4 \mathrm{~mm}$. Thus, if the pupil is less than $4 \mathrm{~mm}$, less light will be available for analysis, and noise will increase. The blue light image in the fovea (lowest fundus AF) may reach levels of nonlinear behavior with increased noise levels. The green light 
image and more peripheral sites at both wavelengths will be less affected because the AF is larger, hence leading to the observed underestimation of MPOD and variation in degree of underestimation across its spatial profile. On the basis of these outcomes, we would suggest that future studies consider implementation of a standardized methodological approach to MPOD measurement using dual-wavelength AF. This approach should include sufficient mydriasis to allow a minimum pupil size greater than $4 \mathrm{~mm}$ under intense bright light stimulation. The methodological approach should also be more comprehensively described. Additionally, the reporting of MPOD should include a quality metric such as the SD values reported herein, and consideration given to the rejection of MPOD values that do not meet SD criteria. In our study, for minimum pupil size under bright illumination conditions of over $4 \mathrm{~mm}$, SD values at $0.23^{\circ}$ eccentricity ranged from 0.04 to 0.1 . Using the linear regression equation, the 0.13 predicted SD value at $0.23^{\circ}$ eccentricity for a pupil size of $4 \mathrm{~mm}$ may provide a reasonable cut-off value for acceptable MPOD measurement quality (SD values were typically lower more peripherally).

\section{Limitations}

One of the limitations of this study is that pupil size and MPOD are measured using two different devices and there was a short time gap between conducting pupillometry and measuring MPOD. The light intensity in the Spectralis device is higher than that used in the Aladdin device, so that the minimum pupil size during MPOD measurement could be slightly smaller than that recorded using Aladdin. Another limitation is the relatively small sample size, as the undilated measurement could be obtained only for 18 subjects. However, the sample size was sufficient to show highly statistically significant differences between pre- and post-dilation MPOD and SD values across the spatial profile of MP, and allowed the estimation of the suggested cut-off point for pupil size and quality of measurement. One final possible limitation relates to the use of $0.5 \%$ tropicamide as the mydriatic agent. Most studies that have reported the type of mydriatic agent used have employed tropicamide plus phenylephrine, or if used alone, a more potent $1 \%$ tropicamide concentration. Although it might be perceived as a limitation, the use of the lower $0.5 \%$ concentration herein was deliberate for two reasons. First, the lower concentration offers benefits in terms of the lesser and shorter duration quality of life impact on study participants and patients in clinical practice. Second, and more importantly, it was implemented as a means to determine whether any effects observed might relate to the degree of mydriasis induced. The use of the $0.5 \%$ concentration resulted in a broad range of postdilation pupil size from just under $4 \mathrm{~mm}$ to over $7 \mathrm{~mm}$. It was felt that a higher concentration may cause a greater average increase in pupil size but narrower overall range. Figure 4 demonstrates very clearly the absence of any relationship between the measurement SD and pupil size post-dilation despite the more than $3 \mathrm{~mm}$ range of pupil size. As such, this study demonstrates that measurement quality is essentially independent of the type of mydriatic used once sufficient mydriasis is induced to yield a minimum photopic pupil diameter of no less than $4.35 \mathrm{~mm}$.
Of note, pupil size in the current study was measured using an automated pupillometer. However, it has been shown that there is no significant disagreement between manual and automated pupillometer measurements, so the suggestions for pupil size presented in the current study can be applicable to both automated and manual pupil size measurements. ${ }^{42}$

\section{Conclusions}

In conclusion, this study confirms that pupillary dilation is required for accurate measurement of MPOD when using the dual-wavelength AF technique, and reinforces that the measurement protocol should include confirmation that pupil size exceeds the entrance pupil size of the device (in this case $4 \mathrm{~mm}$ ) under intense bright light stimulation. Although the Heidelberg Spectralis device does not provide a quantitative indicator for the MP measurement quality, SD values at each retinal eccentricity indicative of image quality can be extracted from the graphical output. Our data indicate that a cut-off SD value at $0.23^{\circ}$ retinal eccentricity of 0.11 may provide a simple clinical guide to ensure optimal image quality. Numerous published studies do not report using mydriatic eye drops to dilate the pupil before MPOD measurement, or omit specific detail such as the actual pupil size following dilation with this technique. ${ }^{24,27-31}$ Future publications should, therefore, provide more specific detail on the treatment of pupils and the resultant quality metrics obtained in the respective studies to allow more complete and accurate assessment of the reported findings.

\section{Declaration of interest}

The authors report no conflicts of interest and have no proprietary interest in any of the materials mentioned in this article.

\section{Funding}

This work was supported by the Technological University Dublin "Fiosraigh" Dean of Graduate Research School Award. The funders had no role in study design, data collection, and analysis, decision to publish, or preparation of the manuscript.

\section{ORCID}

Ekaterina Loskutova (D) http://orcid.org/0000-0002-2438-9036

\section{References}

1. Loskutova E, Nolan J, Howard A, Beatty S. Macular pigment and its contribution to vision. Nutrients. 2013;5:1962-69.

2. Loughman J, Davison P, Akkali M, Nolan J, Beatty S. Macular pigment and its contribution to visual performance and experience. J Optom. 2010;3:73-89.

3. Vishwanathan R, Schalch W, Johnson EJ. Macular pigment carotenoids in the retina and occipital cortex are related in humans. Nutr Neurosci. 2016;19:95-101.

4. Craft NE, Haitema TB, Garnett KM, Fitch KA, Dorey CK. Carotenoid, tocopherol, and retinol concentrations in elderly human brain. J Nutr Health Aging. 2004;8:156-62.

5. Tanprasertsuk J, Mohn ES, Matthan NR, Lichtenstein AH, Barger K, Vishwanathan R, Johnson MA, Poon LW, Johnson EJ. Serum carotenoids, tocopherols, total n-3 polyunsaturated fatty 
acids, and n-6/n-3 polyunsaturated fatty acid ratio reflect brain concentrations in a cohort of centenarians. Journals Gerontol Ser A. 2019;74(3):306-314.

6. Vishwanathan R, Kuchan MJ, Sen S, Johnson EJ. Lutein and preterm infants with decreased concentrations of brain carotenoids. J Pediatr Gastroenterol Nutr. 2014;59:659-65.

7. Hammond BR, Johnson EJ, Russell RM, Krinsky NI, Yeum KJ, Edwards RB, Snodderly DM. Dietary modification of human macular pigment density. Invest Ophthalmol Vis Sci. 1997;38:1795-801.

8. Landrum JT, Bone RA, Joa H, Kilburn MD, Moore LL, Sprague KE. A one year study of the macular pigment: the effect of 140 days of a lutein supplement. Exp Eye Res. 1997;65:57-62.

9. Siah WF, Loughman J, O'Brien C. Lower macular pigment optical density in foveal-involved glaucoma. Ophthalmology. 2015;122:2029-37.

10. Arunkumar R, Calvo CM, Conrady CD, Bernstein PS. What do we know about the macular pigment in AMD: the past, the present, and the future. Eye. 2018;32:992-1004.

11. Scanlon G, Connell P, Ratzlaff M, Foerg B, McCartney D, Murphy A, O'Connor K, Loughman J. Macular pigment optical density is lower in type 2 diabetes, compared with type 1 diabetes and normal controls. Retina. 2015;35:1808-16.

12. Johnson EJ. Role of lutein and zeaxanthin in visual and cognitive function throughout the lifespan. Nutr Rev. 2014;72:605-12.

13. Vishwanathan R, Iannaccone A, Scott TM, Kritchevsky SB, Jennings BJ, Carboni G, Forma G, Satterfield S, Harris T, Johnson KC, et al. Macular pigment optical density is related to cognitive function in older people. Age Ageing. 2014;43:271-75.

14. Loskutova E, Shah K, Flitcroft ID, Setti A, Butler JS, Nolan Y, Paudel N, Loughman J. Lutein and zeaxanthin: the possible contribution, mechanisms of action and implications of modern dietary intake for cognitive development in children. HRB Open Res. 2019;2:8.

15. Stringham JM, Hammond BR, Nolan JM, Wooten BR, Mammen A, Smollon W, Snodderly DM. The utility of using customized heterochromatic flicker photometry (cHFP) to measure macular pigment in patients with age-related macular degeneration. Exp Eye Res. 2008;87:445-53.

16. Wooten BR, Hammond BR, Land RI, Snodderly DM. A practical method for measuring macular pigment optical density. Invest Ophthalmol Vis Sci. 1999;40:2481-89.

17. Siah WF, O'Brien C, Loughman JJ. Macular pigment is associated with glare-affected visual function and central visual field loss in glaucoma. Br J Ophthalmol. 2018;102:929-35.

18. Delori FC. Autofluorescence method to measure macular pigment optical densities fluorometry and autofluorescence imaging. Arch Biochem Biophys. 2004;430:156-62.

19. Dennison JL, Stack J, Beatty S, Nolan JM. Concordance of macular pigment measurements obtained using customized heterochromatic flicker photometry, dual-wavelength autofluorescence, and single-wavelength reflectance. Exp Eye Res. 2013;116:190-98.

20. Delori FC, Goger DG, Hammond BR, Snodderly DM, Burns SA. Macular pigment density measured by autofluorescence spectrometry: comparison with reflectometry and heterochromatic flicker photometry. J Opt Soc Am A. 2001;18:1212.

21. Conrady CD, Bell JP, Besch BM, Gorusupudi A, Farnsworth K, Ermakov I, Sharifzadeh M, Ermakova M, Gellermann W, Bernstein PS. Correlations between macular, skin, and serum carotenoids. Invest Ophthalmol Vis Sci. 2017;58:3616-27.

22. Obana A, Gohto Y, Sasano H, Gellermann W, Sharifzadeh M, Seto T, Bernstein PS. Grade of cataract and its influence on measurement of macular pigment optical density using autofluorescence imaging. Invest Ophthalmol Vis Sci. 2018;59:3011-19.

23. Akuffo KO, Nolan JM, Stack J, Power R, Kirwan C, Moran R, Corcoran L, Owens N, Beatty S. The impact of cataract, and its surgical removal, on measures of macular pigment using the heidelberg spectralis HRA+OCT multicolor device. Investig Opthalmology Vis Sci. 2016;57:2552.

24. Crosby-Nwaobi R, Hykin P, Peto T, Sivaprasad S. An exploratory study evaluating the effects of macular carotenoid supplementation in various retinal diseases. Clin Ophthalmol. 2016;10:835.
25. Zeimer MB, Krömer I, Spital G, Lommatzsch A, Pauleikhoff D. Macular telangiectasia: patterns of distribution of macular pigment and response to supplementation. Retina. 2010;30:1282-93.

26. Hammond CJ, Liew SHM, Van Kuijk FJ, Beatty S, Nolan JM, Spector TD, Gilbert CE. The heritability of macular response to supplemental lutein and zeaxanthin: a classic twin study. Invest Ophthalmol Vis Sci. 2012;53:4963-68.

27. Meyer Zu Westrup V, Dietzel M, Pauleikhoff D, Hense HW. The association of retinal structure and macular pigment distribution. Investig Opthalmology Vis Sci. 2014;55:1169.

28. Daga FB, Ogata NG, Medeiros FA, Moran R, Morris J, Zangwill LM, Weinreb RN, Nolan JM. Macular pigment and visual function in patients with glaucoma: the San Diego macular pigment study. Invest Ophthalmol Vis Sci. 2018;59:4471-76.

29. Nolan JM, Loskutova E, Howard A, Mulcahy R, Moran R, Stack J, Bolger M, Coen RF, Dennison J, Akuffo KO, et al. The impact of supplemental macular carotenoids in Alzheimer's disease: a randomized clinical trial. J Alzheimers Dis. 2015;44:1157-69.

30. Nolan JM, Loskutova E, Howard AN, Moran R, Mulcahy R, Stack J, Bolger M, Dennison J, Akuffo KO, Owens N, et al. Macular pigment, visual function, and macular disease among subjects with Alzheimer's disease: an exploratory study. J Alzheimers Dis. 2014;42:1191-202.

31. Helb H-M, Charbel Issa P, VAN DER Veen RLP, Berendschot TTJM, Scholl HPN, Holz FG. Abnormal macular pigment distribution in type 2 idiopathic macular telangiectasia. Retina. 2008;28::808-16.

32. Wüstemeyer H, Jahn C, Nestler A, Barth T, Wolf S. A new instrument for the quantification of macular pigment density: first results in patients with AMD and healthy subjects. Graefe's Arch Clin Exp Ophthalmol. 2002;240:666-71.

33. Jahn C, Wüstemeyer H, Brinkmann C, Trautmann S, Mössner A, Wolf S. Macular pigment density in age-related maculopathy. Graefes Arch Clin Exp Ophthalmol. 2005;243:222-27.

34. Lima VC, Rosen RB, Maia M, Prata TS, Dorairaj S, Farah ME, Sallum J. Macular pigment optical density measured by dual-wavelength autofluorescence imaging in diabetic and nondiabetic patients: a comparative study. Investig Opthalmology Vis Sci. 2010;51:5840.

35. Obana A, Gellermann W, Gohto Y, Seto T, Sasano H, Tanito M, Okazaki S. Reliability of a two-wavelength autofluorescence technique by Heidelberg Spectralis to measure macular pigment optical density in Asian subjects. Exp Eye Res. 2018;168:100-06.

36. Dietzel M, Zeimer M, Heimes B, Pauleikhoff D, Hense H-W. The ringlike structure of macular pigment in age-related maculopathy: results from the muenster aging and retina study (MARS). Investig Opthalmology Vis Sci. 2011;52:8016.

37. Zeimer M, Dietzel M, Hense HW, Heimes B, Austermann U, Pauleikhoff D. Profiles of macular pigment optical density and their changes following supplemental lutein and zeaxanthin: new results from the LUNA study. Investig Opthalmology Vis Sci. 2012;53:4852.

38. R Core Team I. R: A language and environment for statistical computing. R Foundation for Statistical Computing, Vienna: R Foundation for Statistical Computing Authors R Core Team. 2014.

39. Howells O, Eperjesi F, Bartlett H. Measuring macular pigment optical density in vivo: a review of techniques. Graefe's Arch Clin Exp Ophthalmol. 2011;249:315-47.

40. Trieschmann M, Spital G, Lommatzsch A, van Kuijk E, Fitzke F, Bird AC, Pauleikhoff D. Macular pigment: quantitative analysis on autofluorescence images. Graefe's Arch Clin Exp Ophthalmol. 2003;241:1006-12.

41. Ctori I, Mahroo OA, Williams KM, Hammond CJ, Huntjens B. Repeatability of the macular pigment spatial profile: A comparison of objective versus subjective classification. Acta Ophthalmol. 2018;96:e797-e803.

42. Smith J, Flower O, Tracey A, Johnson P. A comparison of manual pupil examination versus an automated pupillometer in a specialised neurosciences intensive care unit. Aust Crit Care. 2020;33:162-66. 